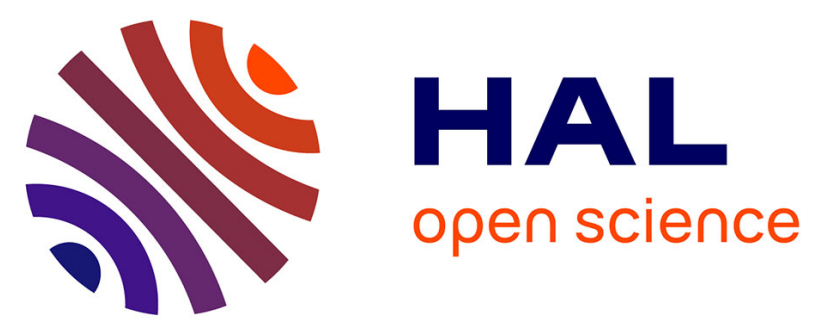

\title{
DECONVOLUTION REGULARIZED USING FUZZY C-MEANS ALGORITHM FOR BIOMEDICAL IMAGE DEBLURRING AND SEGMENTATION
}

Benoît Lelandais, Frederic Duconge

\section{- To cite this version:}

Benoît Lelandais, Frederic Duconge. DECONVOLUTION REGULARIZED USING FUZZY CMEANS ALGORITHM FOR BIOMEDICAL IMAGE DEBLURRING AND SEGMENTATION. International Symposium on BIOMEDICAL IMAGING (ISBI): From Nano to Macro, Apr 2015, NewYork, France. cea-01155379

HAL Id: cea-01155379

https://hal-cea.archives-ouvertes.fr/cea-01155379

Submitted on 26 May 2015

HAL is a multi-disciplinary open access archive for the deposit and dissemination of scientific research documents, whether they are published or not. The documents may come from teaching and research institutions in France or abroad, or from public or private research centers.
L'archive ouverte pluridisciplinaire HAL, est destinée au dépôt et à la diffusion de documents scientifiques de niveau recherche, publiés ou non, émanant des établissements d'enseignement et de recherche français ou étrangers, des laboratoires publics ou privés. 


\title{
DECONVOLUTION REGULARIZED USING FUZZY C-MEANS ALGORITHM FOR BIOMEDICAL IMAGE DEBLURRING AND SEGMENTATION
}

\author{
Benoît Lelandais, Fréderic Ducongé \\ CEA, I²BM, MIRCen - CNRS URA2210, LMN - Université Paris Sud \\ 18 route du Panorama, 92260 Fontenay-aux-Roses, France
}

\begin{abstract}
We address deconvolution and segmentation of blurry images. We propose to use Fuzzy C-Means (FCM) for regularizing Maximum Likelihood Expectation Maximization deconvolution approach. Regularization is performed by focusing the intensity of voxels around cluster centroids during deconvolution process. It is used to deconvolve extremely blurry images. It allows us retrieving sharp edges without impacting small structures. Thanks to FCM, by specifying the desired number of clusters, heterogeneities are taken into account and segmentation can be performed. Our method is evaluated on both simulated and Fluorescence Diffuse Optical Tomography biomedical blurry images. Results show our method is well designed for segmenting extremely blurry images, and outperforms the Total Variation regularization approach. Moreover, we demonstrate it is well suited for image quantification.
\end{abstract}

Index Terms - Deconvolution, segmentation, deblurring, regularization, Fuzzy C-Means, heterogeneity, quantification, molecular imaging

\section{INTRODUCTION}

In biomedical imaging, the blurring of images is often a problem for the segmentation and the quantification of the signal. To solve this problem, deconvolution is used [1]. Because deconvolution is an ill-posed problem, there is no consensus on this issue. In fluorescence imaging, the well-known Maximum Likelihood Expectation Maximization (MLEM) deconvolution method, is often used because it has the advantage to ensure non-negativity [2]. However, this iterative process has the drawback to converge to noise producing ringing artifacts. Convergence is ensured if regularization is implemented [1]. In biomedical imaging, Wavelet

To appear in Proc. ISBI2015, April 1619, 2015

(C)2015 IEEE. Personal use of this material is permitted. Permission from IEEE must be obtained for all other uses, in any current or future media, including reprinting/republishing this material for advertising or promotional purposes, creating new collective works, for resale or redistribution to servers or lists, or reuse of any copyrighted component of this work in other works.
$[3,4]$ and Total Variation [5, 6, 7] are widely used for regularization. Multiscale wavelet-based approach reduces the noise in the residual using Bayeshrink filtering. Because of the multiscale approach, small structures can be preserved. However, contours are not sharp [4]. Total Variation enforces smoothness in the convolved data by adding a term based on spatial context (L1 or L2 norm) [5]. Using L1 norm, the advantage is that contours are sharp. The disadvantage is that small structures can be removed.

The Fuzzy C-Means (FCM) [8] clustering method is widely used for biomedical image segmentation. Derived from fuzzy logic [9], FCM is used to model imprecision by affecting to each voxel membership degrees according to multiple clusters. In [10], the authors propose to use FCM for dealing with heterogeneity in magnetic resonance images. In [11], a wavelet based approach is incorporated to the FCM algorithm in order to model heterogeneity using a multiscale approach in positron emission tomography images. In these two approaches, a spatial constraint is also added for noisy data regularization. Using FCM in imaging, incorporating neighborhood information is needed to improve voxel labeling. Although it can be easy to use neighborhood to remove noise, it is more difficult to remove blur corresponding to a lack of knowledge.

Methods combining both restoration and segmentation have been proposed. In [12], a bayesian framework is used for dealing with both noise and blurry information. In [13], the Mumford and shah model is presented. The method consists of the minimization of a sum of criterions whose aim is to perform image restoration with homogeneous regions and thin boundaries.

Here, we propose a new regularization approach for MLEM deconvolution using FCM. The idea is to focus the intensity of voxels around cluster centroids during deconvolution process. Instead of using neighborhood information, regularization is performed according to a specified number of clusters that is given by the experimenter. The advantage is that small structures are preserved and sharp edges are retrieved. Moreover the image is segmented.

The paper is organized as follow. First, some background about deconvolution and FCM are given. It is followed by our proposed method which is then evaluated on simulated im- 
ages and on images acquired from fluorescence Diffuse Optical Tomography in vivo molecular imaging system.

\section{BACKGROUND}

\subsection{Deconvolution}

The general framework in deconvolution is based on the following model:

$$
i(x)=(o \otimes p)(x)+n(x),
$$

where $i$ denotes the recored image, $o$ denotes the real object, $p$ is the Point Spread Function (PSF), $n$ is an additive noise and $\otimes$ is the convolution operator. MLEM deconvolution consists in reconstructing $o$ by finding $o=\arg \max _{o}\{p(i \mid o)\}$. Because of the quantum nature of light, photon noise is Poisson distributed. Thus, $o$ maximizes the likelihood function:

$$
p(i \mid o)=\prod_{x} \frac{(p \otimes o)(x)^{i(x)} \cdot \exp (-(p \otimes o)(x))}{i(x) !}
$$

or minimizes the negative log-likelihood function given by:

$$
J_{M L}=\sum_{x}-i(x) \ln (p \otimes o)(x)+(p \otimes o)(x)+\ln (i(x) !)
$$

Assuming that $\sum_{x} p(x)=1$, finding the null derivative of (3) according to $o(x)$ leads to the following updating:

$$
o^{t+1}(x)=o^{t}(x) \frac{i(x)}{\left(p \otimes o^{t}\right)(x)} \otimes p(-x)
$$

where $p(-x)$ corresponds to the mirrored PSF function. As mentioned in introduction, this update scheme has the drawback to amplify noise, that is why regularization is needed [1]. Regularization can be performed using L1 norm Total Variation (TV1) denoising approach [5]. The functional becomes:

$$
J_{M L_{T V 1}}=J_{M L}+\lambda_{T V 1} \sum_{x}|\nabla o(x)|
$$

where $\nabla$ is the gradient operator, and $\lambda_{T V 1}$ is a parameter controlling the regularization term. It leads to the updating:

$$
o^{t+1}(x)=\frac{o^{t}(x) \frac{i(x)}{\left(p \otimes o^{t}\right)(x)} \otimes p(-x)}{1-\lambda_{T V 1} \operatorname{div}\left(\frac{\nabla o(x)}{|\nabla o(x)|}\right)}
$$

where div is the divergence operator.

\subsection{Fuzzy C-Means (FCM)}

FCM [8] is a clustering method allowing us to assign an object to two or more clusters with different membership degrees. Let $j=\{1, \ldots, C\}$, where $C$ is the number of cluster, FCM is based on the minimization of the objective function:

$$
J_{F C M}=\sum_{x} \sum_{j}^{C} \mu_{j}^{m}(x)\left\|i(x)-c_{j}\right\|^{2}
$$

with the constraint $\sum_{j} \mu_{j}=1$, where $\mu_{j}(x)$ is the membership degree of $i(x)$ according to cluster $j$, and where $\left\|i(x)-c_{j}\right\|$ is the norm expressing the similarity between $i(x)$ and the the cluster centroid $c_{j} . m>1$ is a parameter controlling the fuzziness of the data ( $m=2$ by default). A large $m$ value results in fuzzy clusters, whereas a value close to 1 results in crisp clusters. Finding the null derivative of (7) according to $c_{j}$ and $\mu_{j}(x)$ leads to the following alternately updating:

$$
\begin{gathered}
c_{j}=\frac{\sum_{x} \mu_{j}^{m}(x) i(x)}{\sum_{x} \mu_{j}^{m}(x)} \quad \forall j=\{1, \ldots, C\} \\
\mu_{j}(x)=\frac{1}{\sum_{k}\left(\frac{\left\|i(x)-c_{j}\right\|}{\left\|i(x)-c_{k}\right\|}\right)^{\frac{2}{m-1}}} \quad \forall j=\{1, \ldots, C\}
\end{gathered}
$$

Derived from fuzzy logic [9], FCM can be used to model heterogeneity in biomedical imaging $[10,11]$.

\section{PROPOSED METHOD}

We propose to use FCM for regularizing the MLEM deconvolution. It is performed by minimizing both the MLEM and the FCM objective functions. The resulting functional is:

$$
J_{M L_{F C M}}=J_{M L}+\lambda_{F C M} \sum_{j}^{C} \mu_{j}^{m}(x)\left\|o(x)-c_{j}\right\|^{2}
$$

subject to $\sum_{j} \mu_{j}=1$, where $\lambda_{F C M}$ controls the regularization term. By finding the null derivation of (10) according to $c_{j}$, $\mu_{j}(x)$ and $o(x)$, we adopt the following alternately updating:

$$
\begin{gathered}
c_{j}=\frac{\sum_{x} \mu_{j}^{m}(x) o^{t}(x)}{\sum_{x} \mu_{j}^{m}(x)} \quad \forall j=\{1, \ldots, C\} \\
\mu_{j}(x)=\frac{1}{\sum_{k}\left(\frac{\left\|o^{t}(x)-c_{j}\right\|}{\left\|o^{t}(x)-c_{k}\right\|}\right)^{\frac{2}{m-1}}} \quad \forall j=\{1, \ldots, C\} \\
o^{t+1}(x)=\frac{o^{t}(x)\left(\frac{i(x)}{\left(p \otimes o^{t}\right)(x)} \otimes p(-x)\right)}{1+\lambda_{F C M} 2 \sum_{j}^{C} \mu_{j}^{m}(x)\left(o^{t}(x)-c_{j}\right)}
\end{gathered}
$$

Because the intensities of voxels are brought close to the cluster centroid they belong to, noise is not amplified during the deconvolution process. As in FCM algorithm, $m$ controls the fuzziness of the data. Furthermore, because the membership degrees are in the power of $m$ in equation (13), a large value of $m$ results in a low regularization for data whose intensity is far from cluster centroids. We set $m$ to its default value $m=2$. As using TV1 approach, the regularization is controlled by a parameter $\lambda_{F C M}$. Because the regularization term in equation 13 is according to $\left(o^{t}(x)-c_{j}\right)$ that depends on image content, we suggest to normalize $\lambda_{F C M}$ according to the maximum image intensity: $\lambda_{F C M}=\frac{\Lambda_{F C M}}{\max _{x}(i(x))}$. Our proposed method performs simultaneously the segmentation by specifying the number of clusters, and the deconvolution with a regularization approach focusing the intensity of voxels around clusters. 


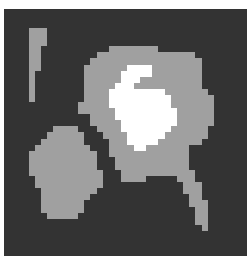

(a) Simulated image

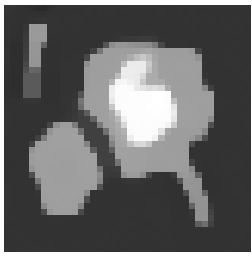

(d) $\mathrm{ML}_{\mathrm{TV} 1}$

$R M S D=1.67 E^{-3}$

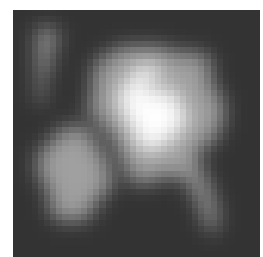

(b) Blurry image

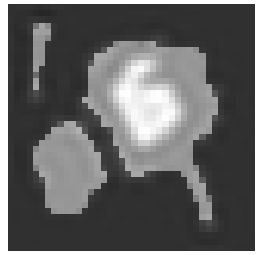

(e) $\mathrm{ML}_{\mathrm{FCM} 2}$

$R M S D=2.26 E^{-3}$

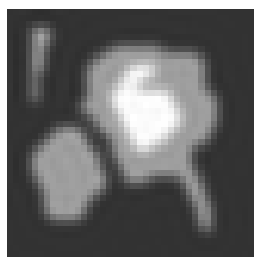

(c) $\mathrm{ML}$ $R M S D=4.04 E^{-3}$

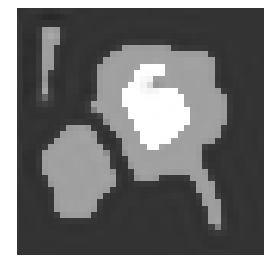

(f) $\mathrm{ML}_{\mathrm{FCM}} 3$ $R M S D=0.20 E^{-3}$
Fig. 1. Deconvolution results on a simulated blurry image using MLEM with and without regularization approaches (TV1 and FCM). The RMSD are given below each figure.

\section{EVALUATION}

\subsection{Simulated image}

To evaluate the behavior of our deconvolution method, a simulated image given in Figure 1(a) was used. It consists in three gray scales $(0.2,0.63$ and 1$)$ and three regions: one thin, one heterogeneous and one contaminating another. The image is blurred using a Gaussian PSF whose standard deviation is 1.6 pixels (see Figure 1(b)). The same PSF is used for deconvolution. Three deconvolution methods were compared, the MLEM method (ML), the MLEM regularized using TV1 (ML $\left.\mathrm{MV}_{1}\right)$ and our proposed method using two and three classes (respectively $\mathrm{ML}_{\mathrm{FCM} 2}$ and $\mathrm{ML}_{\mathrm{FCM}}$ ). Iterations are perform until the normalized sum of image differences between two iterations is lower than $10 E^{-5}$. For our method, centroids are initialized using C-Means algorithm, and $o(x)^{0}$ is set to $i(x)$. Regularization parameter values were determined empirically, by applying the deconvolutions with different parameter values and by selecting those minimizing the criterions (equations (5) and (7)). We obtained $\lambda_{T V 1}=0.001$ and $\Lambda_{F C M}=0.001$. Root-Mean-Square Deviations (RMSD) between the deconvolution results and the simulated image (Figure 1(a)) are used for evaluation.

The deconvolution results are given in Figure 1. The best result is obtained with $\mathrm{ML}_{\mathrm{FCM} 3}$ presenting the lower RMSD. The contours are sharp, the small region is retrieved and the contamination is well corrected. By maximizing the membership value of each pixel according to the three classes, a segmented image can be obtained. We observed that only 2 pixels were not well classified with $\mathrm{ML}_{\mathrm{FCM} 3}$ : in the thin and the white regions. With ML, the contours are still blurry and the signal vary slightly inside homogeneous regions. Using $\mathrm{ML}_{\mathrm{TV} 1}$, the contours are sharp and noise is not amplified. However the small region is not well retrieved and the pixel values between the two contaminated regions is higher than in simulated image ( 0.25 instead of 0.2$)$. Concerning $\mathrm{ML}_{\mathrm{FCM} 2}$, we observe that two classes of pixels (black and gray) have been regularized, whereas the contours of the third class (White pixels) are still blurry as using ML. This result is interesting because it shows that our method can be applied in the case of heterogeneous regions, affecting to the voxels different membership degrees according to the clusters. In conclusion, as long as the number of classes is known, the best deconvolution is obtained using our method. In biomedical cases, this number if often known by the experimenter thanks to its expertise in a pathology. Furthermore, the estimation of this number is still possible, using for example Bayesian Information Criterion as proposed in [11].

\section{2. fluorescence Diffuse Optical Tomography (fDOT) im- ages}

In order to validate our method on biomedical images, we used images provided by fDOT in a mouse. It is a new imaging technique used to follow in 3-Dimensions (3D) a fluorescent probe in tissues [14]. Reconstructed images are extremely blurry, leading to imprecision that corresponds to a lack of knowledge [15]. It is the case at the contour of objects, and especially in the presence of small structures. In medical applications as oncology, a precise segmentation is necessary in order to quantify the quantity of probes in tumors. Due to imprecision, fDOT image segmentation is difficult to achieve and measurements can not reflect the actual probe quantity in tissues.

The images are obtained as follow. First, a transparent capillary filled with $4 \mu \mathrm{L}$ of fluorescent probes was inserted under the skin of a mouse (see Figure 2(a)). Then, a transillumination $685 \mathrm{~nm}$-laser scan was performed at the region of interest (see Figure 2(a)), and 3D reconstruction was performed [14]. Figure 2(c) gives an example of reconstructed image. Voxels after reconstruction are $0.6 \times 0.6 \times 1 \mathrm{~mm}^{3}$. By changing the quantity of probes in the capillary, five quantities with the same volume were imaged: 2.6, 10.6, 21.2, 42.4 and $84.8 \mathrm{pMol}$. Assuming the autofluorescence of the mouse is null compared to the probes, the images give information about the total quantity of fluorescent signal in probes. Four segmentation methods are compared: a thresholding with a 0 threshold that allows us to measure the total signal quantity (Thresh), the FCM using 2 classes (FCM2), the ML $\mathrm{TV}_{\mathrm{T} 1}$ deconvolution followed by FCM2 $\left(\mathrm{ML}_{\mathrm{TV} 1}^{\prime}\right)$ and our proposed method with two classes $\left(\mathrm{ML}_{\mathrm{FCM} 2}\right) . \lambda_{T V 1}$ and $\Lambda_{F C M}$ was set to 0.1 , allowing us to obtain sharp contours without noise amplification. Moreover, increasing these parameter values increases the size of the fluorescent region. Concerning $\mathrm{ML}_{\mathrm{FCM} 2}$ the value was chosen in order to obtain fluorescent 


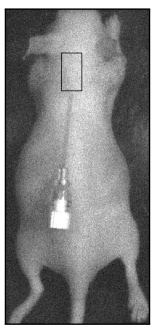

(a)

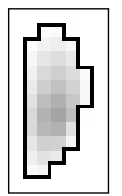

(c) Thresh

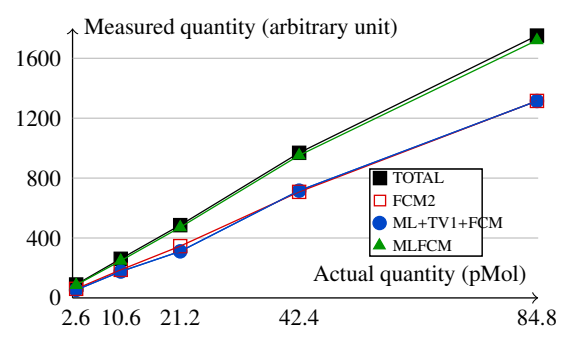

(b)

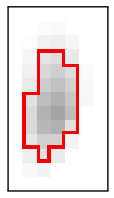

(d) FCM2

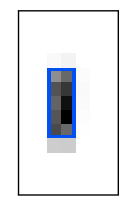

(e) $\mathrm{ML}_{\mathrm{TV} 1^{\prime}}$

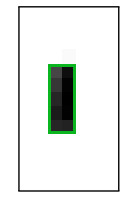

(f)MLFCM2
Fig. 2. Segmentation of a fDOT image using four methods. (a) is a white planar image of a capillary inserted in a mouse. The fluorescent scanned region is represented by a rectangle. (b) is a comparison of the measured quantity of fluorescence with each method according to each capillary. (c), (d), (e) and (f) correspond to fluorescence segmentation results on one slice according to the four compared methods.

volumes around $12 \mu \mathrm{L}$. This volume corresponds to only 27 voxels. A Gaussian PSF is used whose 3D standard deviation is $\sim 1 \mathrm{~mm}$. This value was approximated using a reconstructed image of a capillary filled with a probe of $0.5 \mu \mathrm{L}$, which is close to the volume of a voxel $(0.44 \mu \mathrm{L})$. Finally, using FCM, segmentation results are obtained by choosing the class having the highest membership degree.

Figure 2 gives the segmentation results according to the four compared methods. Thresh allows retrieving the total quantity of signal. However, the volume of capillaries is about $108.2 \pm 23.1 \mu \mathrm{L}$ that is extremely large compared to the actual filled volume. Using FCM segmentation method, the volume is also large $(41.2 \pm 2.7 \mu \mathrm{L})$. Moreover, as we can see in Figure 2(b), the measured quantity corresponds to an under-estimation of the total quantity given by Thresh. The large measured volumes using Thresh and FCM2 are due to blurry signal in fDOT images. $\mathrm{ML}_{\mathrm{TV} 1}^{\prime}$ and $\mathrm{ML}_{\mathrm{FCM} 2}$ deconvolution methods give the best volume estimation (respectively $11.6 \pm 1.5 \mu \mathrm{L}$ and $12.2 \pm 1.2 \mu \mathrm{L}$ ). Moreover, the deconvolved image using $\mathrm{ML}_{\mathrm{FCM} 2}$ is sharper than using $\mathrm{ML}_{\mathrm{TV} 1}^{\prime}$ (see Figure 2(e) vs. (f)). Thanks to our proposed regularization using FCM, intensity of voxels is close to the centroid of the cluster it belongs to, resulting in sharp contours. Furthermore, we observe that the measured quantity is under-estimated using $\mathrm{ML}_{\mathrm{TV} 1}^{\prime}$, but is well estimated using $\mathrm{ML}_{\mathrm{FCM} 2}$ (see Figure 2(b)). In conclusion, our method gives the best volume estimation without under-estimation of the quantity of probes in the segmented region.

\section{CONCLUSION}

Dedicated to blurry image segmentation, we propose to use FCM for regularization of MLEM deconvolution method. Regularization is achieved focusing the intensity of voxels close to the centroid of the cluster it belongs to. Furthermore, it allows us to deal with heterogeneity by affecting to each voxel membership degrees according to the different clusters. Applied on simulated and fDOT images, the results show our method is well designed for segmenting extremely blurry images, and outperforms the TV1 regularization approach.

Our method removes blurry information corresponding to imprecision thanks to the knowledge about the number of clusters. It can be applied on blurry biomedical images. For dealing with both imprecision and uncertainty due to noise, our method could also integrate TV1 regularization.

In future works, the PSF of fDOT images will be estimated more accurately. The noise will also be estimated and the robustness to our method toward this noise will be evaluated. The automatic determination of the cluster number in each region to segment will also be studied.

\section{REFERENCES}

[1] P. Pankajakshan, G. Engler, L. Blanc-Féraud, and J. Zerubia, "Deconvolution and denoising for confocal microscopy," in Modeling in Computational Biology and Biomedicine. Springer-Verlag, 2013.

[2] P. Sarder and A. Nehorai, "Deconvolution methods for 3-D fluorescence microscopy images," Signal Processing Magazine, vol. 23, no. 3, pp. 32-45, 2006.

[3] C. Chaux, L. Blanc-Féraud, and J. Zerubia, "Waveletbased restoration methods: application to $3 \mathrm{~d}$ confocal microscopy images," in Proc. SPIE, 2007, vol. 6701, pp. 67010E-67010E-11.

[4] N. Boussion, C. Cheze Le Rest, M. Hatt, and D. Visvikis, "Incorporation of wavelet-based denoising in iterative deconvolution for partial volume correction in whole-body PET imaging," Eur. J. Nucl. Med. Mol. Imaging, vol. 36, no. 7, pp. 1064-1075, Jul 2009.

[5] N. Dey, L. Blanc-Feraud, C. Zimmer, P. Roux, Z. Kam, J.C. Olivo-Marin, and J. Zerubia, "Richardson-Lucy algorithm with total variation regularization for 3D confocal microscope deconvolution," Microsc. Res. Tech., vol. 69, no. 4, pp. 260-266, Apr 2006.

[6] M. Laasmaa, M. Vendelin, and P. Peterson, "Application of regularized Richardson-Lucy algorithm for deconvolution of confocal microscopy images," J Microsc, vol. 243, no. 2, pp. 124-140, Aug 2011. 
[7] Margret K., Thorsten S., M. Temerinac-Ott, J. Padeken, P. Heun, O. Ronneberger, and T. Brox, "Blind deconvolution of widefield fluorescence microscopic data by regularization of the optical transfer function (otf)," in Conference on Computer Vision and Pattern Recognition (CVPR). 2013, pp. 2179-2186, IEEE.

[8] J.C. Bezdek, Pattern Recognition with Fuzzy Objective Function Algorithms, Kluwer Academic Publishers, Norwell, MA, USA, 1981.

[9] L.A. Zadeh, "Fuzzy sets," Information Control, vol. 8, pp. 338-353, 1965.

[10] M.N. Ahmed, S.M. Yamany, N. Mohamed, A.A. Farag, and T. Moriarty, "A modified fuzzy C-means algorithm for bias field estimation and segmentation of MRI data," IEEE Trans Med Imaging, vol. 21, no. 3, pp. 193-199, Mar 2002.

[11] S. Belhassen and H. Zaidi, "A novel fuzzy C-means algorithm for unsupervised heterogeneous tumor quantification in PET," Med Phys, vol. 37, no. 3, pp. 13091324, Mar 2010.

[12] H. Ayasso and A. Mohammad-Djafari, "Joint NDT image restoration and segmentation using Gauss-MarkovPotts prior models and variational Bayesian computation," IEEE Trans Image Process, vol. 19, no. 9, pp. 2265-2277, Sep 2010.

[13] L. Bar, T.F. Chan, G. Chung, M. Jung, N. Kiryati, R. Mohieddine, N. Sochen, and L.A. Vese, "Mumford and shah model and its applications to image segmentation and image restoration," in Handbook of Mathematical Methods in Imaging, 2011, pp. 1095-1157.

[14] A. Koenig, L. Hervé, J. Boutet, M. Berger, J.M. Dinten, A.D. Silva, P. Pelti, and P. Rizo, "Fluorescence diffuse optical tomographic system for arbitrary shaped small animals.," in International Symposium on Biomedical Imaging (ISBI). 2008, pp. 1593-1596, IEEE.

[15] A. Garofalakis, A. Dubois, B. Kuhnast, D. M. Dupont, I. Janssens, N. Mackiewicz, F. Dolle, B. Tavitian, and F. Duconge, "In vivo validation of free-space fluorescence tomography using nuclear imaging," Optics Letters, vol. 35, no. 18, pp. 3024-3026, Sep 2010. 